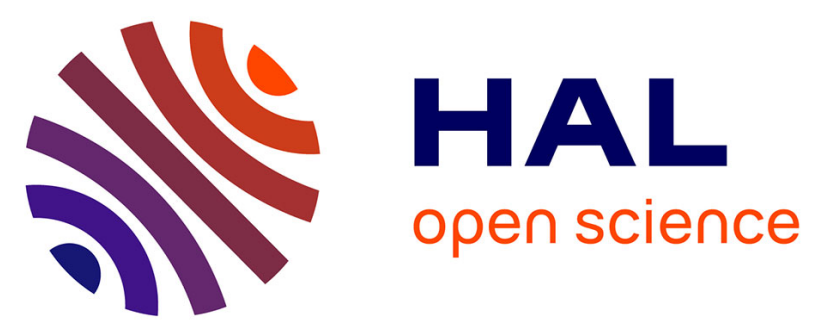

\title{
Enhanced Photochromism of Diarylethene Induced by Excitation of Localized Surface Plasmon Resonance on Regular Arrays of Gold Nanoparticles
}

Ryohei Yasukuni, Nordin Félidj, Leïla Boubekeur-Lecaque, Stéphanie Lau-truong, Jean Aubard

\section{To cite this version:}

Ryohei Yasukuni, Nordin Félidj, Leïla Boubekeur-Lecaque, Stéphanie Lau-truong, Jean Aubard. Enhanced Photochromism of Diarylethene Induced by Excitation of Localized Surface Plasmon Resonance on Regular Arrays of Gold Nanoparticles. ChemPhysChem, 2020, 21 (22), pp.2614-2619. 10.1002/cphc.202000613 . hal-03036870

\section{HAL Id: hal-03036870 https://hal.science/hal-03036870}

Submitted on 9 Dec 2020

HAL is a multi-disciplinary open access archive for the deposit and dissemination of scientific research documents, whether they are published or not. The documents may come from teaching and research institutions in France or abroad, or from public or private research centers.
L'archive ouverte pluridisciplinaire HAL, est destinée au dépôt et à la diffusion de documents scientifiques de niveau recherche, publiés ou non, émanant des établissements d'enseignement et de recherche français ou étrangers, des laboratoires publics ou privés. 
1

\title{
Enhanced Photochromism of Diarylethene Induced by Excitation of Localized Surface Plasmon Resonance on Regular Arrays of Gold
}

\section{Nanoparticles}

\author{
Ryohei Yasukuni, ${ }^{[a, b]}$ Nordin Félidj, ${ }^{*[a]}$ Leïla Boubekeur-Lecaque, ${ }^{[a]}$ Stéphanie Lau- \\ Truong $^{[\mathrm{a}]}$ and Jean Aubard ${ }^{[\mathrm{a}]^{* *}}$ \\ ${ }^{a}$ Dr. R. Yasukuni \\ Present address: Graduate School of Science and Technology, Nara Institute of Science and Technology, \\ Ikoma, 8916-5, Japan; \\ ${ }^{b}$ Dr. R. Yasukuni, Prof. N. Félidj, Dr. L. Boubekeur-Lecaque, S. Lau-Truong, Prof. J. Aubard \\ Université de Paris, ITODYS, CNRS, UMR 7086, 15 rue J-A de Baïf, F-75013 Paris, France \\ Corresponding authors: \\ *Prof.Nordin.Félidj: nordin.felidj@univ-paris-diderot.fr \\ **Jean Aubard: jean.aubard@ @niv-paris-diderot.fr
}

\begin{abstract}
Localized surface plasmon resonance (LSPR) excitation on the photochromic reaction of a diarylethene derivative (DE) was studied by surface enhanced Raman scattering (SERS). UV and visible light irradiations transform reversibly DE between open-form (OF) and closed-form (CF) isomers, respectively. A mixture of PMMA and DE (either OF or CF isomer) was spin-coated onto gold nanorods (GNRs) arrays, designed by electron beam lithography, with two localized surface plasmon resonances (LSPR) at distinct wavelengths, due to their anisotropy. The photochromic reaction rates from CF to OF isomers, under LSPR excitation, were monitored from SERS spectral changes under different polarizations, on the same GNR substrate to compare the effect of LSPR field strength. It appears that the photoisomerization rate was faster when LSPR was excited with the polarization parallel to the GNR long axis. The present results highlight a potential genuine mechanism, from near field LSPR excitation, involved in the photochromic enhancement of diarylethene photochromes.
\end{abstract}




\section{Introduction}

Photochromism of organic compounds is defined as a reversible transformation induced by light between two isomeric states with different molecular absorption bands. ${ }^{[1]}$ Among various series of organic photochromes, diarylethenes (DE) have received much interest during the past 20 years thanks to their interesting photochromic properties. These molecules show good efficiency of the photoisomerization process, both in solution and in solid states, high fatigue resistance, thermal stability and fast conversion between isomeric states, which makes DE promising candidates for optical devices such as single molecular level optical switches and memories. ${ }^{[2]}$ The use of DE in practical applications requires however, that more efficient photochromism takes place, at least in one direction and this requires an enhancement of the corresponding photo-induced quantum yield. ${ }^{[3]}$ Excitation of localized surface plasmon (LSP) resonance of metal nanostructures can be one possibility to induce efficient photoisomerization.

The excitation of localized surface plasmon (LSP) resonances, which arise from collective oscillations of free electrons at the surface of noble metal nanoparticles (NPs), leads to intense extinction (absorption and scattering) bands, mostly in the visible and near -infrared, the spectral position of which depends on the NP size, shape, chemical nature and local dielectric environment. In addition, under LSP excitation, the local electromagnetic (EM) field is strongly enhanced at the surface of nanostructures. ${ }^{[4]}$ The localized EM field nearby metal nanoparticles (NPs), mainly Au and Ag, also leads to giant scattering and emission processes such as Surface Enhanced Raman Scattering (SERS) and Surface Enhanced Fluorescence (SEF) which have nowadays various applications in the fields of (bio)sensor and chemical analysis. ${ }^{[5]}$ On the other hand, coupling plasmonic nanoparticles with photochromic molecules could lead, under certain conditions, to an improvement of the photoswitching efficiency. ${ }^{[6]}$ The distance between the metallic NPs and the photochromic molecules, and the overlap between the LSP resonance and the electronic transition of the photochromic unit have been shown to play a major role. Coupling LSPR to molecular resonance induces modifications either on the molecular properties or on the optical properties of plasmonic nanostructures and these modifications can be detected by SERS or by far field extinction spectroscopy. It should be noted that efficient coupling occurs, if and only if an optimal distance, molecule/ NPs, is fulfilled and when LSPR and molecular resonances overlap spectrally. Lastly, if the molecule is light sensitive, e.g. a photochromic molecule, LSPR excitation could lead to enhanced 
photochromism. In this latter case, several reports have shown that photoisomerization kinetics were accelerated (enhanced) in the presence of gold or silver nanoparticles with respect to the solution photochromism. ${ }^{[7]}$ In these reports, authors claimed that the photoisomerization rate was increased due to enhanced EM field following LSPR excitation. Various effects in the presence of metal (gold and silver) nanoparticles could take place, such as nonlinear processes (e.g. two photon process) after light excitation, photo-thermal isomerization due to local heating following nonradiative plasmon energy relaxation, charge transfer between molecules and metals, and they should be taken into account since they could contribute to enhance the isomerization efficiency on the plasmonic substrate. ${ }^{[8]}$ In most cases, however, they can hardly be distinguished from the effect of LSPR excitation. Moreover, in previous studies, the photoisomerization process was monitored by UV-Visible absorption change which is not sensitive enough when analyses at the nanoscale are considered. In addition, such detection methods could induce some isomerization of the photochromic state under study, which is not relevant for an efficient analysis of the genuine mechanism. In an effort to design LSPR based efficient photochromic switching systems, the mechanism of photochromic enhancement on metal nanoparticles has to be clarified.

In this work, the effect of LSPR excitation on the photochromic reaction of a diarylethene derivative (DE) was studied by surface enhanced Raman scattering (SERS). DE photochromes, either open or closed isomers (resp. OF or $\mathrm{CF}$ ), were deposited by spin coating onto gold nanorods (GNRs) regular arrays. GNRs arrays were designed by Electron Beam Lithography (EBL) and display two LSPR modes along the GNR short (S) and long (L) axis, respectively. These modes can be selectively excited by switching the excitation light polarization. The main advantage of using such EBL structures is the possibility to tune in a fine way the LSPR wavelengths. Moreover, the EBL fabricated array the metal surface of the NPs is free of any chemical species, and therefore a chemical reactivity between DE isomers and the metal surface is unlikely, in contrast to colloidal NPs. This rule out a contribution by a charge transfer mechanism of the observed enhanced ring opening photochromic reaction. Photoisomerization kinetics from CF to OF isomers was monitored from SERS intensity changes under the two different polarizations, using $785 \mathrm{~nm}$ laser excitation. By this way, it has been possible to analyze the contribution of the different processes, arising from near field LSPR excitation, on the enhanced photochromism of DE.

\section{Experimental}


Synthesis of 1, 2-bis(5'-ethoxy-2'-(2"-pyridyl)thiazolyl)perfluorocyclopentene (DE) and the characterization of its optical properties have already been reported in detail elsewhere. ${ }^{[3]}$

Plasmonic substrates consisted of gold nanorod arrays were designed by electron beam lithography. In the fabrication process, a $100 \mathrm{~nm}$ thick layer of poly(methyl methacrylate), PMMA, was spin-casted onto a glass/indium tin oxide (ITO, $80 \mathrm{~nm}$ thickness) substrate. After chemical development of the exposed areas, thermal evaporation of gold and a lift-off procedure followed. The particle height was set at $40 \mathrm{~nm}$. The whole arrays area was fixed to $100 \times 100 \mu \mathrm{m}^{2}$. The electron beam lithography has the advantage of the fine control of the shape and the spatial arrangement of NPs, allowing the tunability of the LSP resonances which depend on the polarization of the incident light.

For SERS experiments the studied photochrome (DE), either OF or CF isomers, was dispersed in a polymer matrix and spin-coated onto the GNRs array in order to obtain uniform and reproducible samples. ${ }^{[9]}$ For that purpose, a stock solution obtained by diluting twice the $0.02 \mathrm{M}$ of DE in chlorobenzene containing 3 wt\% poly(methyl methacrylate) (PMMA, Allresist, AR-P 671.02) was prepared. A drop (20 $\mu \mathrm{l})$ of the solution was deposited on the GNRs array and then spread by spin-coating at $4000 \mathrm{rpm}$ for $60 \mathrm{~s}$. Prior to spin-coating, DE in the chlorobenzene-PMMA solution was transformed into the colored state (CF isomer) by UV light irradiation for 10 min using a spot light source (Hamamatsu, Lightningcure, LC8, equipped with a $150 \mathrm{~W}$ Xe-Hg lamp) through a NIR long-pass filter and a UV band-pass filter leading to a large amount of CF and weak thermo and photodegradation. ${ }^{[9]}$

Raman spectra were recorded with a commercial confocal Raman microspectrometer (Horiba Jobin-Yvon, Labram HR 800) equipped with a thermoelectric cooled CCD and a motorized XY displacement stage. A continuous wave (CW) laser diode at $\lambda=785 \mathrm{~nm}$ (Sacher Lasertechnik, RL-785-1008-0153) was used both for NIR SERS excitation and CF to OF photoisomerisation in the NIR. The laser was focused onto samples through a microscope 50x objective (Olympus, NA: 0.75), and Raman scattering was collected through the same objective. Incident laser polarizations were switched with a $\lambda / 2$ plate and the laser power was adjusted to $30 \mu \mathrm{W}$ at the sample position with a power meter (Coherent, laser checker). 
Extinction spectra of GNRAs were recorded with a spectrometer (LOT ORIEL, 74050 model, 300-1000 nm spectral range) equipped with a CCD camera (ANDOR, CCD-8855) under an upright optical microscope (Olympus, BX51TF) through a 50× objective (Olympus, NA: 0.7).

\section{Results and Discussion}

In Fig. 1(a), the photochromic reaction of the DE molecule is illustrated: upon UV irradiation the open-ring isomer ( $\mathrm{OF}$, uncolored) photoisomerizes to the closed-ring isomer ( $\mathrm{CF}$, colored). After irradiation with visible light the colored isomer totally bleaches indicating a complete back reaction from CF to OF isomer. ${ }^{[9]}$ It should be noted that the color change induced by UV irradiation in the diarylethene family is ascribed to a photocyclisation process, which leads to a more extended $\pi$-electron conjugation. ${ }^{[10]}$

UV-visible absorption spectra of OF and CF isomers of DE in toluene are shown in Fig. 1(b). The open form has an absorption maximum at $c a .350 \mathrm{~nm}$; UV irradiation transforms OF into CF isomer which presents a broad visible absorption band in the $450-730 \mathrm{~nm}$ range $(\lambda \max$ ca. 580-600 $\mathrm{nm}$ ) that disappears under visible light irradiation. Fig. 1(c) shows the experimental Raman spectra of DE for CF and OF isomers in the solid state. In Raman experiments, to avoid strong photoisomerization induced with a visible laser line, NIR excitation at 785

nm was used. ${ }^{[9]}$ The spectral changes observed between Raman spectra excited at $785 \mathrm{~nm}$ and recorded before and after UV irradiation (Fig. 1c) have been unambiguously assigned to OF and CF isomers. ${ }^{[10]}$ 
FIG. 1 (a) Photochromic reaction scheme of 1, 2-bis(5'-ethoxy-2'-(2"-pyridyl)thiazolyl)perfluorocyclopentene (DE) after UV and visible light irradiations. (b) UV-VIS absorption spectra of OF and CF in toluene; c) Experimental Raman spectra of DE in the solid state, for CF and OF isomers, excited at $785 \mathrm{~nm}$. Copyright 2013 John Wiley \& Sons, Ltd. ${ }^{[10]]}$

A scanning electron microscope image of an EBL fabricated GNR array and its extinction spectrum are shown in Fig. 2. As observed in the insert, the size and shape of nanorods were uniform within the whole array. In the extinction spectrum two bands were observed at $550 \mathrm{~nm}$ and $674 \mathrm{~nm}$; these bands correspond to LSPR modes in air along the short (S) and long (L) axes of the nanorods, the dimensions of which are respectively, 50 and $100 \mathrm{~nm}$ for a $40 \mathrm{~nm}$ height (Fig 2a, insert). These modes can be selectively excited using a polarized incident light along S or L directions as shown in Fig. 2b (red continuous lines). Deposition onto the GNRs array of a thin PMMA layer containing the DE photochrome, in either OF or CF state (see experimental procedure above in the text), led to a red shift of extinction bands, by more than $60 \mathrm{~nm}$ for that corresponding to the LSPR mode L (Fig. 2b, red dashed lines). It is noteworthy that this red-shift is accompanied by a small increase of the LSPR bandwidth for the mode 
$\mathrm{L}$, due to higher values of the imaginary part of the dielectric constant of the metal around $700 \mathrm{~nm}$. This LSPR red shift is due to an increase of the surrounding refractive index by the PMMA layer deposited onto gold nanorods. This red-shift is much more significant for $\mathrm{L}$ polarization than for S polarization, due to a stronger sensitivity of the LSPR, with respect to the surrounding medium, towards the red spectral range. ${ }^{[11]]}$

(a)
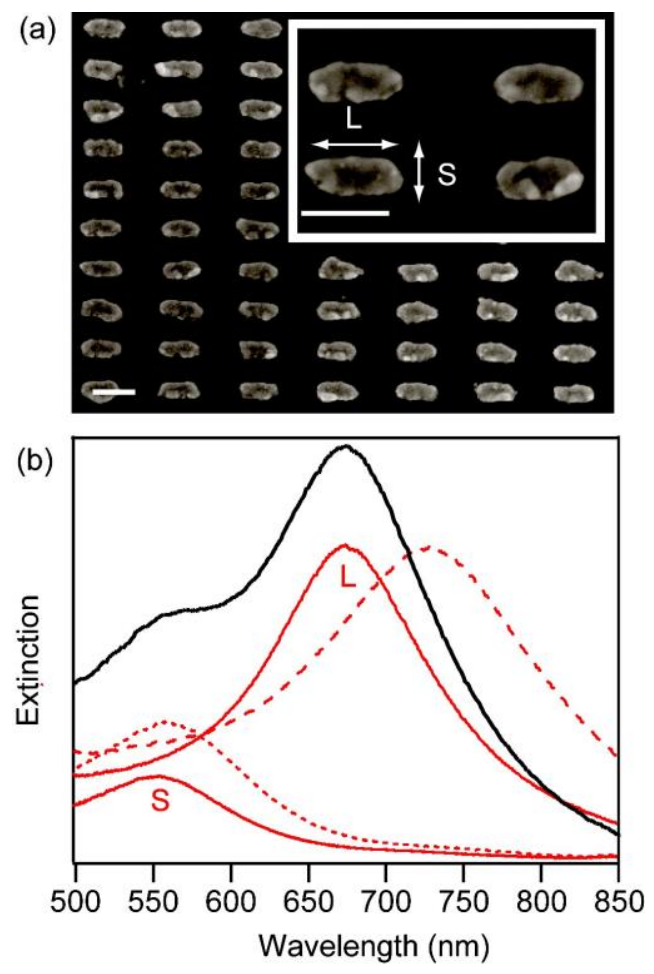

FIG. 2 (a) SEM image of a GNRA fabricated on an ITO substrate by electron beam lithography. S and L indicate the polarizations along the short and long axe of GNRs, respectively. The scale bar represents $100 \mathrm{~nm}$; (b) Extinction spectra of the GNR array in air, illuminated with unpolarized light (black solid line), L- and Spolarized lights (red continuous lines), and after PMMA coating with L- and S-polarized lights (red dashed lines).

SERS spectra of CF isomer on GNR, excited at $785 \mathrm{~nm}$ for $\mathrm{L}$ and $\mathrm{S}$ polarizations are reported in Fig. 3. The SERS spectrum observed for the L polarization (the most intense, see discussion below) is similar to the one recorded previously on gold nanoparticle aggregates. ${ }^{[9]}$ Thus, in the $1300-1700 \mathrm{~cm}^{-1}$ range, the characteristic Raman lines of CF, at $c a .1439,1459$ and $1479 \mathrm{~cm}^{-1}$ (medium to strong) along with the weak lines at $c a$. 1586 and $1616 \mathrm{~cm}^{-1}$, are evidenced (see also Fig. 1c). 


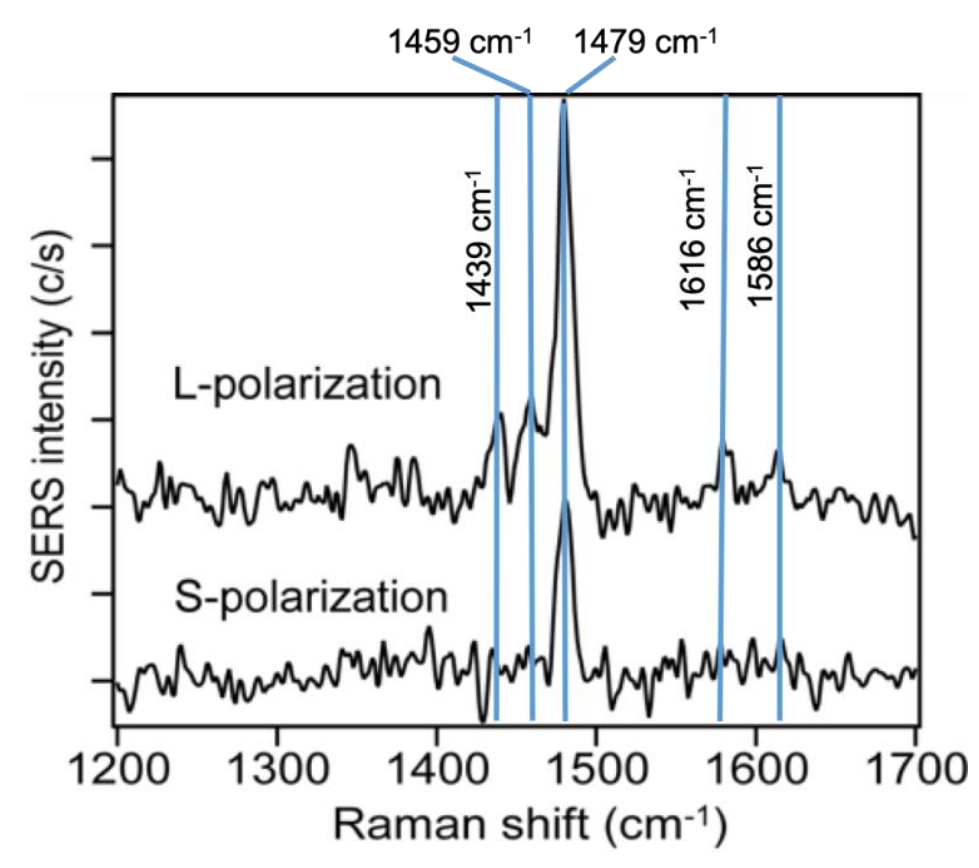

FIG 3. Representative SERS spectra of CF on a GNRs array excited with L- and S-polarizations at $785 \mathrm{~nm}$ (30 $\mu \mathrm{W})$. Background is corrected and baseline is off-set for clarity.

From the detailed vibrational analysis of the SERS spectrum of CF isomer, based on density functional theory (DFT) calculations reported previously ${ }^{[10]}$ these Raman lines arise essentially from vibrational modes of the chromophores, i.e. from the vibrations involved in the extended conjugated system of the closed-ring isomer (CF). Moreover, it was observed that intensity changes of the most intense line at $1479 \mathrm{~cm}^{-1}$ can be directly related to the presence (or not) of the CF isomer, i.e. to DE photoisomerization from OF to CF and vice-versa. ${ }^{[9]}$ Indeed, SERS experiments were performed previously to check that the intensity decrease of the $1479 \mathrm{~cm}^{-1}$ SERS line under $785 \mathrm{~nm}$ laser excitation ( $30 \mu \mathrm{W}$ laser power) was due to photoisomerization from CF to OF isomers and not to photodegradation (fig 4). ${ }^{[9]}$ Briefly, in these experiments, a set of SERS spectra was recorded after a series of visible $(633 \mathrm{~nm})$ and UV-blue $(405 \mathrm{~nm})$ laser irradiations, as it appears in Figure 4. For $633 \mathrm{~nm}$ and $405 \mathrm{~nm}$ we used the same objective and the same sample positions as the ones during $785 \mathrm{~nm}$ measurements. As observed in Figure 4, the intensity of the $1479 \mathrm{~cm}^{-1}$ SERS line, characteristic of CF isomer, strongly decreases after visible laser irradiation but almost the total intensity of this line was recover after UV-blue laser irradiation. This 
indicates that no critical photodegradation occurred and these cyclic SERS spectral changes recorded with the 785 $\mathrm{nm}$ laser excitation at $30 \mu \mathrm{W}$ power may be safely associated with the CF to OF photoisomerization of DE. However, no Raman line corresponding to $\mathrm{OF}$ isomer appears during the ring-opening process induced in this case by visible laser irradiation. It is likely that this could be due to the much weaker Raman cross section at 785 $\mathrm{nm}$ of $\mathrm{OF}$ than $\mathrm{CF}$. Moreover, it was mentioned that the Raman intensity of $\mathrm{CF}$ isomer could be enhanced (with respect to that of $\mathrm{OF}$ isomer) by a (molecular) pre-resonance effect at $785 \mathrm{~nm}$. ${ }^{[9]}$ According to the very low absorbance of CF above $750 \mathrm{~nm}$ (see Fig. 1b), the efficiency of this effect with an excitation at $785 \mathrm{~nm}$ remains however questionable.

It should be noted in Fig. 3 that the SERS spectrum with L-polarization is almost three times higher than that with S-polarization (precisely, $c a .2 .7$, as measured with respect to the $1479 \mathrm{~cm}^{-1}$ integrated line intensity). Since the $785 \mathrm{~nm}$ laser excitation is within the large LSPR band of L-polarization (see Fig. 2b) this ensures, as expected, to stronger SERS enhancement than with S-polarization.

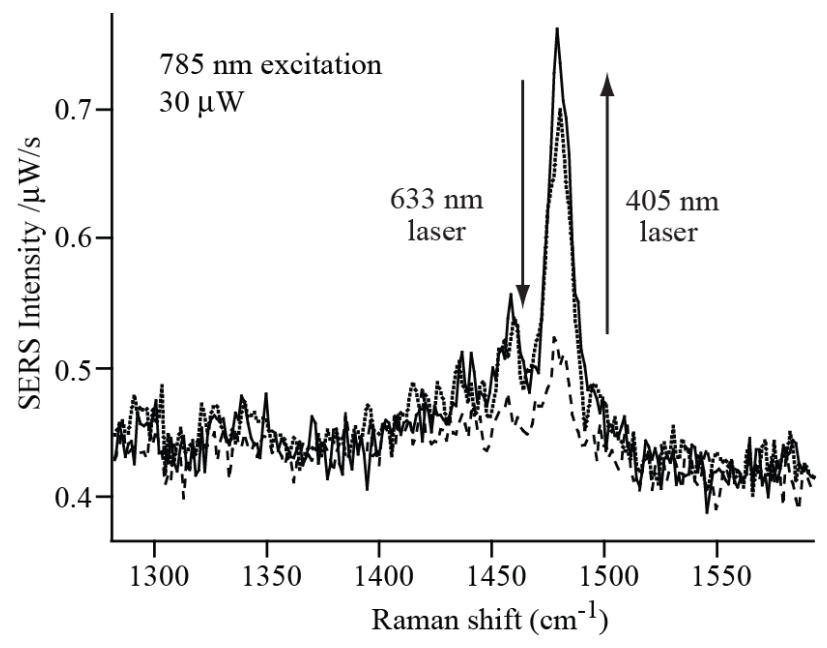

Fig 4. SERS spectral changes on gold spherical nanoparticles, covered with a PMMA film containing CF isomer, excited at $785 \mathrm{~nm}$ in a sequence of laser irradiations. The spectrum in solid line is recorded before any irradiation; the spectrum in dash line has been recorded after an excitation at $633 \mathrm{~nm}$, and the spectrum in dot line after an irradiation at $405 \mathrm{~nm}$ (in the UV-blue region). Arrows indicate directions of the SERS intensity change at 1479 $\mathrm{cm}^{-1}$ after visible (down) and UV (top) irradiation. Copyright 2012, American Chemical Society. ${ }^{[9]}$

In order to understand the origin of the ring-opening isomerization of DE induced by NIR excitation at $785 \mathrm{~nm}$ 
on GNRs, we compared the decrease of the $1479 \mathrm{~cm}^{-1}$ SERS intensity line, on the same substrate, between L- and S-polarizations. Figure 5 displays the decay curves for L- and S-polarizations, obtained after different irradiation times at $785 \mathrm{~nm}$. As it can be seen, the decrease of the $1479 \mathrm{~cm}^{-1}$ SERS intensity line for L and S polarizations proved that photoisomerization occurs in both cases. However, the decay rate is faster in L polarization than in $\mathrm{S}$ polarization. From these experiments we can assume that the isomerization efficiency is enhanced for L polarization on GNRs. To quantify the relative enhancement between L and S polarizations the decay constants were estimated from a simple curve fitting. The decay constants (time constants), $\tau_{\mathrm{L}}=26 \mathrm{~s}$ and $\tau_{\mathrm{S}}=48 \mathrm{~s}$ for L- and S-polarizations respectively, were obtained by a single exponential analysis. Thus, it appears that the ring-opening isomerization rate was almost twice faster (precisely, 1.8) when LSPR was excited at $785 \mathrm{~nm}$ with the polarization parallel to the long axis of GNRs proving that enhanced photochromism takes place by efficient LSPR excitation.

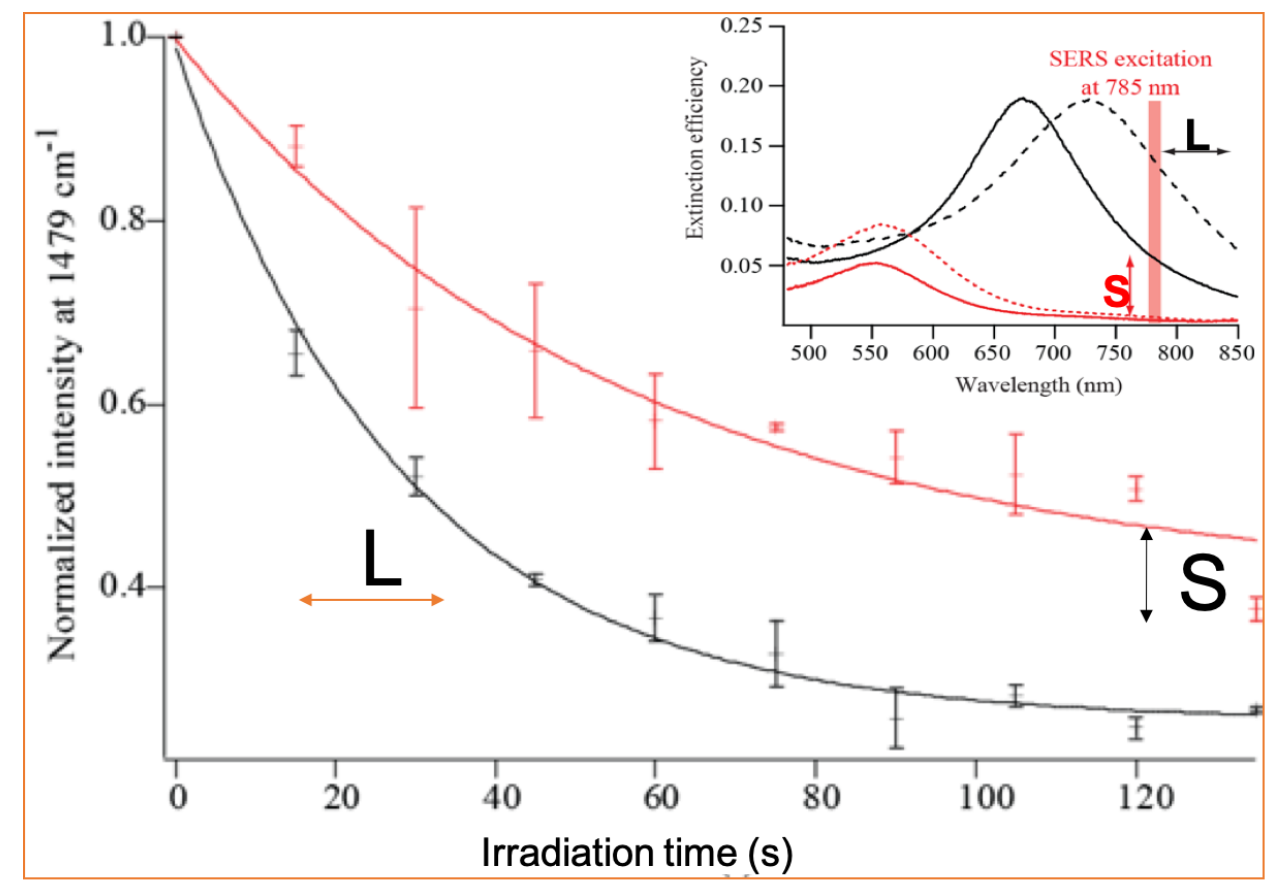

FIG 5. Time decays of the SERS line at $1479 \mathrm{~cm}^{-1}$ excited with L (black) and S (red) polarizations. A series of SERS spectral changes was recorded 5 times at different site of GNRs, and their averaged intensity was normalized and plotted as a function of laser exposure time. The error bars show the standard deviation from 5 measurements. Irradiation time (in sec.) at $785 \mathrm{~nm}$; Insert: Extinction spectra of the GNRs in air, excited with L 
(black) and S (red) polarized lights (continuous lines) and after PMMA coating with L- and S-polarized lights (dashed lines).

Thanks to the SERS intensity decay curves between L and S polarizations, a relative photochromic enhancement factor of $c a .1 .8$ was estimated and this enhancement clearly originated from LSPR excitation. This value must be compared to the ratio of averaged near field strength around GNRs, $\left|E_{L}\right|^{2} /\left|E_{S}\right|^{2}$, where $E_{L}$ and $E_{S}$ are enhanced EM field associated with LSPR excitations with L- and S-polarizations, which can be deduced from the SERS intensity ratio between L- and S-polarizations, $\mathrm{I}_{\mathrm{L}} / \mathrm{I} \mathrm{s}$. Indeed, let us consider a purely electromagnetic (EM) enhancement associated with plasmon excitation in a metal NPs substrate, as in the present SERS experiments on GNRs arrays. In this approach we neglect, on purpose, a possible chemical effect which could occur from an electronic coupling between the adsorbed molecules and the metal surface, through a charge transfer or a bond formation, because it is unlikely and its contribution to enhancement is very weak. Then, if $E(v)$ is the enhanced electric field, evaluated at the molecular positions, that arises when plasmons are excited at frequency $v$, the SERS enhancement factor ( SERS EF) may be approximated by averaging, $[\mathrm{E}(\mathrm{v})]^{4 /}\left[\mathrm{E}_{0}\right]^{4}$, over all the excited molecules, where $\mathrm{E}_{0}$ is the incident (laser) field. It was shown that this $\mathrm{E}^{4}$ rule produces estimations of $\mathrm{EF}$ that are in correct agreement with experimental results. ${ }^{[12]}$ From the above expression of the SERS EF, the $\left|E_{L}\right|^{2} /\left|E_{S}\right|^{2}$ ratio may be estimated from the ratio of SERS intensities between L- and S-polarizations, I $\mathrm{L}_{\mathrm{L}}$ and I s, which are proportional to $\left|E_{L}\right|^{4}$ and $\left|E_{S}\right|^{4}$ respectively. Then, $I_{L} / I_{S}$ may be expressed as $\left|E_{L}\right|^{4} /\left|E_{S}\right|^{4}$. Since the value of $I_{L} / I_{S}$ is ca. 2.7 (see Fig. 3), this leads to ca.1.6, for $\left|E_{L}\right|^{2} /\left|E_{S}\right|^{2}$, in very good agreement with the photochromic enhancement factor of $c a$. 1.8, deduced from the decay curves in Fig 5. This confirms that enhanced photochromism of DE takes place under LSPR excitation.

A question that arises here concerns the surprising small differences observed between SERS intensities for Land S-polarizations, $I_{L}$ and $I_{s}$ (Fig. 3) and hence, between $\left|E_{L}\right|^{2}$ and $\left|E_{S}\right|^{2}$. Indeed, since the $785 \mathrm{~nm}$ laser excitation is within the large extinction band for L-polarization whereas it is totally out of that for S polarization (see Fig. 5, insert), it was expected a huge intensity difference in their respective SERS spectra. This discrepancy, between far field extinction properties and near field SERS intensities demonstrates that LSPR near field properties, as obtained from SERS intensities, play the major role in the photochromic enhancement. These small 
differences observed between SERS intensities for L- and S-polarizations are attributed to the presence of nanoscale surface roughness (NSR) of GNRs in the fabricated EBL arrays. ${ }^{[13]}$ Indeed, it was shown previously that NSR features significantly enhance the SERS intensity of adsorbed probe molecules even when the excitation wavelength lies far from the LSP resonance. ${ }^{[13]}$ Moreover, the NSR that can be efficiently excited in the NIR, does not depend on the excitation polarizations and contribute significantly to the SERS intensity. ${ }^{[13]}$ This could explain the small SERS intensity differences observed between $L$ and S polarization in the present experiments. In order to precise the origin of the enhancement of the ring-opening isomerization one must take into account the various processes that could occur in LSP-enhanced EM fields. Thus, it has been reported that LSPR excitations could lead to multiphoton process, e.g. simultaneous two-photon absorption. ${ }^{[9]}$ However, with the $785 \mathrm{~nm}$ exciting laser line in our experiments, a simultaneous two-photon absorption process ( $\mathrm{ca} .390 \mathrm{~nm}$ ) would lead to a ring-closing isomerization, $(\mathrm{OF} \rightarrow \mathrm{CF})$ of $\mathrm{DE}$ which is opposite to our observed results $(\mathrm{CF} \rightarrow \mathrm{OF})$. Therefore, a simultaneous two-photon absorption cannot explain the observed enhancement of the ring-opening isomerization, $\mathrm{CF} \rightarrow$ OF. Photo thermal effectscould be an alternative mechanism to explain the origin of this enhanced photochromism. ${ }^{[14]}$ Indeed, in SERS experiments, when LSPR are excited, part of the plasmon energy is transformed into heat and the temperature in the vicinity of NPs (mainly at hot-spots or within the gap of coupled NPs) may be high enough to induce thermal isomerization. Although DE isomers, OF and CF, are very stable at room temperature (more than a month), these molecules show however thermal isomerization at high temperature. ${ }^{[15]}$ On the basis of our previous experiments in which thermal ring-opening isomerization of DE deposited onto GNPs aggregates was analyzed by measuring time change of absorption spectra of CF at various temperatures (from 100 to $250{ }^{\circ} \mathrm{C}$ ) it was demonstrated that the thermal isomerization of the studied DE with the $785 \mathrm{~nm}$ exciting laser at a power of $30 \mu \mathrm{W}$ can be ruled out. ${ }^{[9]}$ Another mechanism that could be considered so far to explain the observed photochromism enhancement under LSPR excitation is Plasmon Resonance Energy Transfer (PRET). ${ }^{[16]}$ In PRET the energy is transferred from the plasmon to the molecule. Although a PRET mechanism occurs when an overlap between the LSP resonance and an electronic transition of the molecule under study is observed, it was suggested, above in the main text, that a molecular pre-resonance effect could take place at $785 \mathrm{~nm}$ for the CF isomer; this assumption could be in agreement with a PRET mechanism. ${ }^{[16]}$ 


\section{Conclusion}

We successfully examined the role of near field excitation of LSPR on the photochromism of a diarylethene derivative. Extinction and SERS spectral measurements on gold nanorods (GNRs) arrays confirmed that the EM field strength associated with LSPR excitation could be controlled by switching excitation polarizations on anisotropic GNRs. Photoisomerization kinetics from CF to OF isomers of DE on GNRs was monitored by SERS intensity changes at $785 \mathrm{~nm}$ excitation with polarizations along short (S) and long (L) axes of GNRs. Comparison between $\mathrm{L}$ and $\mathrm{S}$ polarizations showed that the photoisomerization rate was faster when LSPR was efficiently excited with the polarization parallel to the long axis of GNRs ( $c a$. 1.8-fold faster isomerization rate). The ratio of EM field strength between the two excitation polarizations was in good agreement with the photochromic enhancement factor. We also discussed the various processes that could occur in LSP-enhanced EM fields. It appears that the observed photoisomerization enhancement at $785 \mathrm{~nm}$ arises neither from a multiphoton process nor from local heating but we suggest that a plasmon resonance energy transfer (PRET) process could take place. In this respect, the present results highlight, for the first time, a potential genuine mechanism, from near field LSPR excitation, involved in the photochromic enhancement of diarylethene photochromes.

\section{Acknowledgments}

ANR (Agence Nationale de la Recherche) and CGI (Commissariat à l'Investissement d'Avenir) are gratefully acknowledged for their financial support of this work through Labex SEAM (Science and Engineering for Advanced Materials and devices), ANR-10-LABX-096 and ANR-18-IDEX-0001. This work was also supported by the CNRS, and the University of Paris.

\section{Keywords}

Electron beam lithography, Gold nanorods, Localized surface plasmon, Photochromism, SERS. 


\section{References}

[1] a) J. C. Crano, R. J. Guglielmetti, Organic Photochromic and Thermochromic Compounds, Springer US, New York, 1999; b) S. Kobatake, M. Irie Annual Reports Section "C" (Physical Chemistry). 2003, 99, 277-313.

[2] a) K. Matsuda, H. Yamaguchi, T. Sakano, M. Ikeda, N. Tanifuji, M. Irie J. Phys. Chem. C. 2008, 112, 17005-17010; b) H. Tian, Y. L. Feng J. Mater. Chem. 2008, 18, 1617-1622.

[3] A. Spangenberg, R. Metivier, J. Gonzalez, K. Nakatani, P. Yu, M. Giraud, A. Leaustic, R. Guillot, T. Uwada, T. Asahi Adv. Mater. 2009, 21, 309-313.

[4] K. M. Mayer, J. H. Hafner Chem. Rev. 2011, 111, 3828-3857.

[5] a) P. Bharadwaj, P. Anger, L. Novotny Nanotechnology. 2007, 18, 5; b) M. K. Hossain, Y.

Kitahama, G. G. Huang, X. X. Han, Y. Ozaki Anal. Bioanal. Chem. 2009, 394, 1747-1760; c) M. Cottat, C. D'Andrea, R. Yasukuni, N. Malashikhina, R. Grinyte, N. Lidgi-Guigui, B. Fazio, A. Sutton, O.

Oudar, N. Charnaux, V. Pavlov, A. Toma, E. Di Fabnzio, P. G. Gucciardi, M. L. de la Chapelle J. Phys. Chem. C. 2015, 119, 15532-15540.

[6] a) K. Ueno, S. Juodkazis, T. Shibuya, V. Mizeikis, Y. Yokota, H. Misawa J. Phys. Chem. C. 2009, 113, 11720-11724; b) E. Kowalska, O. O. P. Mahaney, R. Abe, B. Ohtani Phys. Chem. Chem. Phys. 2010, 12, 2344-2355.

[7] a) H. Nishi, T. Asahi, S. Kobatake ChemPhysChem. 2012, 13, 3616-3621; b) H. Nishi, T. Asahi, S. Kobatake Phys. Chem. Chem. Phys. 2012, 14, 4898-4905; c) M. L. Ho, B. J. Chi, T. Y. Hung, H. Y. Liao, J. C. Wang, T. Y. Wang, J. J. Shyue Crystengcomm. 2013, 15, 5969-5979.

[8] a) D. A. Alexson, S. C. Badescu, O. J. Glembocki, S. M. Prokes, R. W. Rendell Chem. Phys. Lett. 2009, 477, 144-149; b) S. K. Saikin, R. Olivares-Amaya, D. Rappoport, M. Stopa, A. Aspuru-Guzik Phys. Chem. Chem. Phys. 2009, 11, 9401-9411.

[9] R. Yasukuni, R. Boubekri, J. Grand, N. Felidj, F. Maurel, A. Perrier, R. Metivier, K. Nakatani, P. Yu, J. Aubard J. Phys. Chem. C. 2012, 116, 16063-16069.

[10] R. Boubekri, R. Yasukuni, S. L. Truong, J. Grand, A. Perrier, J. Aubard, F. Maurel J. Raman Spectrosc. 2013, 44, 1777-1785.

[11] H. Gehan, C. Mangeney, J. Aubard, G. Levi, A. Hohenau, J. R. Krenn, E. Lacaze, N. Felidj J. Phys. Chem. Lett. 2011, 2, 926-931.

[12] E. C. Le Ru, E. Blackie, M. Meyer, P. G. Etchegoin J. Phys. Chem. C. 2007, 111, 13794-13803. [13] I. Sow, J. Grand, G. Levi, J. Aubard, N. Felidj, J. C. Tinguely, A. Hohenau, J. R. Krenn J. Phys. Chem. C. 2013, 117, 25650-25658.

[14] a) G. Baffou, Thermoplasmonics: Heating Metal Nanoparticles Using Light, Cambridge University Press, Cambridge, 2017; b) G. Baffou Photoniques. 2018, 42 - 47.

[15] S. Takami, S. Kobatake, T. Kawai, M. Irie Chem. Lett. 2003, 32, 892-893.

[16] Y. H. Choi, T. Kang, L. P. Lee Nano Lett. 2009, 9, 85-90. 